\title{
Akar Kuning (Arcangelisia Flava) As Neuraminidase Inhibitor: Molecular Docking And Pharmacophore Optimization Approach
}

\author{
*Mohammad Rizki Fadhil Pratama \\ Muhammadiyah University of Palangkaraya \\ e-mail: m.rizkifadhil@umpalangkaraya.ac.id
}

\begin{abstract}
Objectives: This study aims to find a relationship between secondary metabolites of akar kuning and neuraminidase (NA) with molecular docking study and also to determine the most potent NA inhibitor from metabolites of akar kuning.

Methods: All ligands were sketched and optimized using Gaussian 03W with Hartree-Fock method basis sets 6-311G. Molecular docking was performed using AutoDock 4.2.6 toward NA in complexes with oseltamivir, a known NA inhibitor as a co-crystal ligand. The main parameter used was the free energy of binding $(\Delta G)$ and dissociation constant $\left(K_{i}\right)$ as affinity marker. Pharmacophore optimization was conducted on metabolite with the highest affinity to assess the main pharmacophore with the highest influence.

Results: Fibleucin provided the most negative $\Delta \mathrm{G}$ and the lowest $\mathrm{K}_{\mathrm{i}}$ toward NA with $-8.12 \mathrm{kcal} / \mathrm{mol}$ and $1.11 \mu \mathrm{M}$, respectively. Further pharmacophore optimization of fibleucin reveals that ether group at position number 25 had the highest influence toward fibleucin affinity and might be considered as the main pharmacophore of fibleucin as NA inhibitor.

Conclusion: in silico molecular docking and pharmacophore optimization results indicated that fibleucin could be considered as NA inhibitor and should be potential to be developed as antiinfluenza particularly to $\mathrm{H} 5 \mathrm{~N} 1$ with oseltamivir resistance.
\end{abstract}

Keywords: Akar kuning, docking, fibleucin, neuraminidase.

\section{INTRODUCTION}

The battle against avian influenza seems to have been won by mankind with the discovery of various neuraminidase (NA) inhibitors, including oseltamivir and zanamivir [1]. Not only fully synthesis compounds, several natural products such as artemisinin derivatives from Artemisia аппиа also proved to have potential to be developed as NA inhibitors [2]. The discovery not only could reduce the resistance of anti-avian influenza drugs but also open up opportunities to optimize the potential of various derivatives from other natural products, especially against oseltamivir resistance avian influenza H5N1 [3].

Akar kuning (Arcangelisia flava), endemic plants from Borneo especially known for have many pharmacology potentials, including as antiviral [4]. Akar kuning was known for containing various bioactive compounds, one of the most famous of which is berberine. However, akar kuning still contains many 
secondary metabolites which potentially have effects as NA inhibitor [5].

Despite have the potential, not every ligand worth to be further researched. The only ligand with good affinity could be further optimized until fit enough to be developed as NA inhibitor [6]. Optimization of the selected ligand can be done in several ways, one of them by pharmacophore modification from test ligand [7]. The discovery of the main pharmacophore with the greatest influence on the affinity of a ligand is performed by deletion of each pharmacophore from a ligand one by one [8]. The modification results then examined with docking method to assess the influence of deleted pharmacophore [9].

In this study, we conducted in silico study to determine the most potent secondary metabolites of akar kuning as NA inhibitor and could be developed on anti-influenza especially $\mathrm{H} 5 \mathrm{~N} 1$ therapy using molecular docking method [2]. We also make pharmacophore modifications of the selected ligand with the highest affinity to determine the main pharmacophore with the highest influence as NA inhibitor.

\section{METHODS AND PROCEDURE}

\section{Preparation of ligands and receptor}

A total of 11 secondary metabolites of akar kuning including 2-dehydroarcangelisinol, 6hydroxyarcangelisin, fibleucin, fibraurin, 6hydroxyfibleucin, 6-hydroxyfibraurin, berberine, columbamine, jatrorrhizine, palmatine, and tinophyllol was selected as test ligands. Structures of all ligands were sketched using GaussView 3.08 software from Gaussian, Inc. all structures were geometry optimized by Hartree-Fock method basis sets 6-311G with Gaussian 03W software from Gaussian, Inc. Geometry optimization provided an ideal conformation of the following compounds in nature [10]. Optimized ligands format changed from .log to .pdb using Open Babel 2.3.2 software [11]. AutoDock 4.2.6 from The Scripps Research Institute was used as docking software [12]. The structure of NA H5N1 in complexes with oseltamivir (protein data bank ID 2HU4) was obtained from website www.rscb.org with the binding site of oseltamivir chosen as active site since this site already known for NAI development [13].

\section{Validation of docking process}

The method used for molecular docking validation was pose selection using co-crystal structure by redocking it into the active site of NA. Thus, redocking was performed with oseltamivir as co-crystal ligand on NA active site [7]. The parameters observed were rootmean-square deviation (RMSD) of co-crystal ligand at the selected binding site [14]. Software for molecular docking is preferred to predict results from experimental poses with RMSD score no more than $2 \AA$. Smaller RMSD score indicates that position of the redocking ligand was closer to crystallography ligand result [15].

\section{Molecular docking studies}


Molecular docking for all test ligands was performed in a similar way as validation process using with similar grid box size and position [16]. The parameter used in docking process was the free energy of binding $(\Delta G)$, dissociation constant $\left(\mathrm{K}_{\mathrm{i}}\right)$, amino acids residues, and number of hydrogen bonds. Ligand affinity toward receptor in docking method was determined by $\Delta \mathrm{G}$ and $\mathrm{K}_{\mathrm{i}}$ scores. More negative $\Delta \mathrm{G}$ and lower $\mathrm{K}_{\mathrm{i}}$ indicated higher ligand affinity toward active site of the receptor [17]. Test ligand with the highest affinity was compared with validation result of co-crystal ligand in the active site to determine the potency of test ligand as NAI [18].

\section{Pharmacophore optimization}

Selected test ligand with the highest affinity toward active site of NA is then pharmacophore optimized to assess

\section{RESULTS}

All test ligands were sketched and geometry optimized as shown in table 1. Redocking result was provided almost at the stacked position with crystallography result (fig.1) with RMSD value $1.989 \AA$ A. Other parameters observed in validation was $\Delta \mathrm{G}, \mathrm{K}_{\mathrm{i}}$, amino acids residues, and numbers of hydrogen bonds of the co-crystal ligand as shown in table 2 .
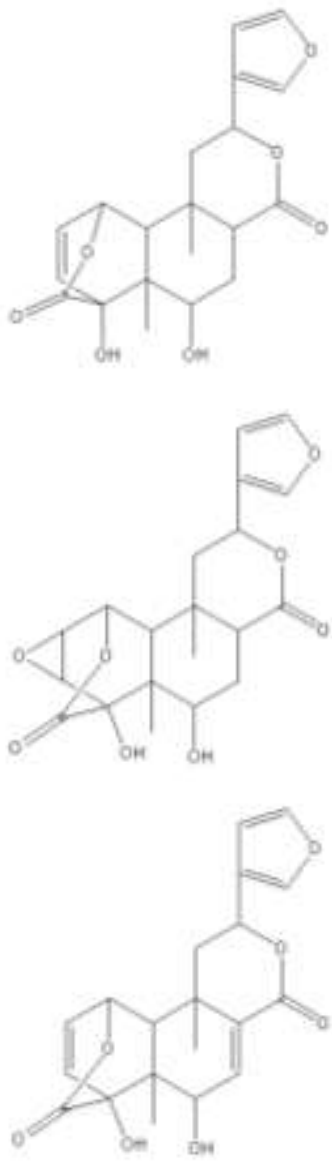
6-hydroxyfibraurin (HFR)

Berberine (BBR)

\section{Columbamine}

(CLB)

Fibleucin

(FLC)

Fibraurin (FRR)

Jatrorrhizine

(JRR)<smiles>CC12CC(c3ccoc3)OC(=O)C1=CC(O)C1(O)C3OC4(OC4C3=O)C21</smiles><smiles>COc1ccc2cc3[n+](cc2c1OC)CCc1cc2c(cc1-3)OCO2</smiles><smiles>COc1cc2c(cc1O)-c1cc3ccc4c(c3c[n+]1CC2)OCCO4</smiles><smiles>CC12CC(c3ccoc3)OC(=O)C1=CC(F)C1(O)C2C=CC12OCCO2</smiles>

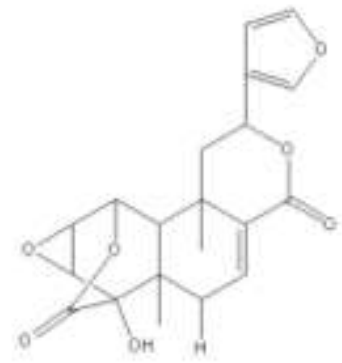<smiles>COc1cc2c(cc1O)CC[n+]1cc3c(OC)c(OC)ccc3cc1-2</smiles> 

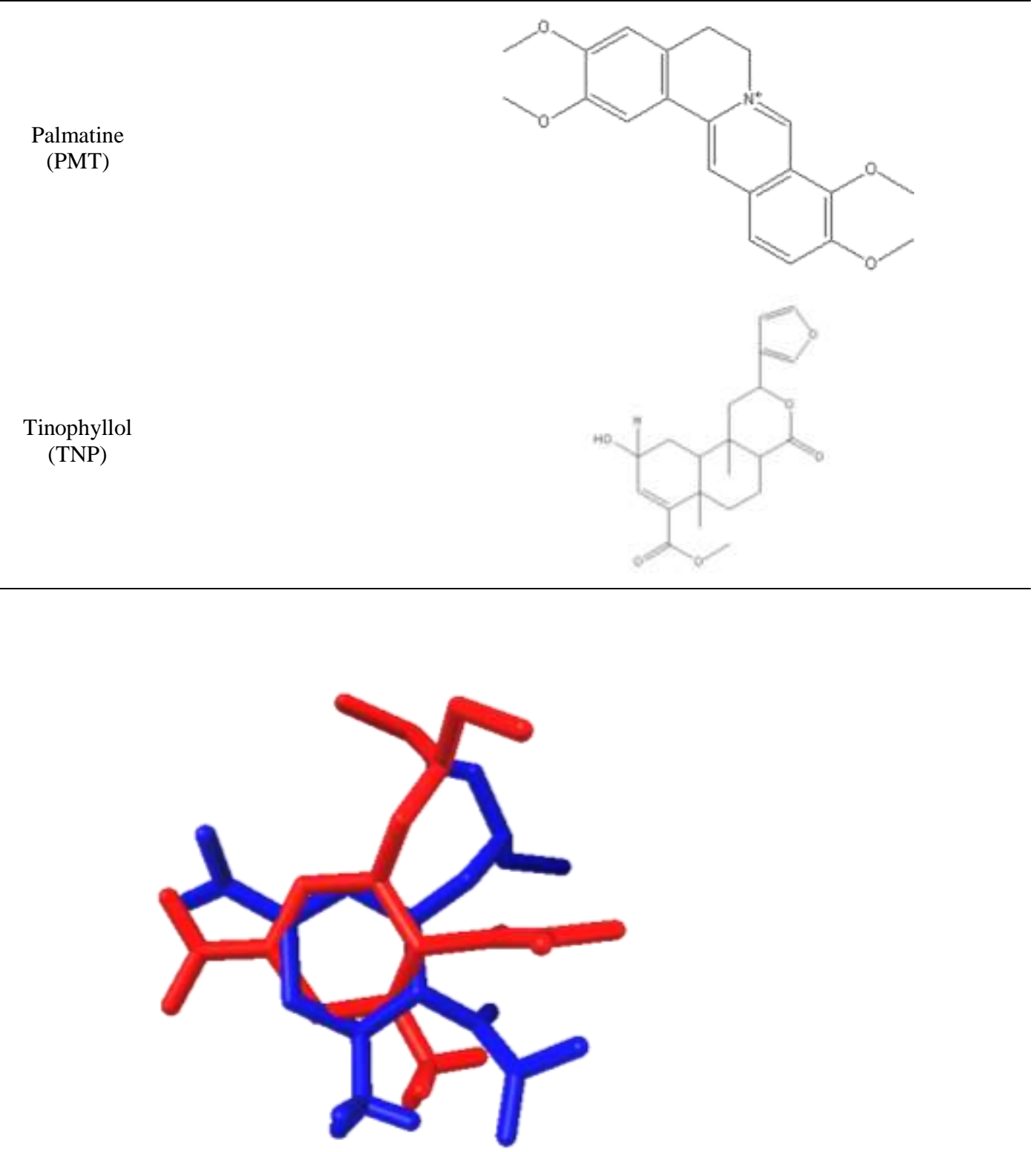

Figure 1. Validation result from NA receptor; RMSD = $1.989 \AA$ (Red: oseltamivir redocking result; blue: oseltamivir crystallography result)

Table 2. Validation result of NA receptors with oseltamivir as co-crystal ligand

\begin{tabular}{ccccccc}
\hline Receptor & Ligand & $\begin{array}{c}\text { RMSD } \\
(\AA)\end{array}$ & $\Delta \mathrm{G}(\mathrm{kcal} / \mathrm{mol})$ & $\mathrm{K}_{\mathrm{i}}(\mu \mathrm{M})$ & Amino acids residues & $\begin{array}{c}\text { Numbers of } \\
\text { hydrogen bonds }\end{array}$ \\
\hline \multirow{2}{*}{ 2HU4 } & & & 119-Glu, 151-Asp, 152-Arg, \\
& Oseltamivir & 1.989 & -9.10 & 0.21376 & $\begin{array}{c}\text { 156-Arg, 178-Trp, 179-Ser, } \\
\text { 224-Arg, 227-Glu, 276-Glu, }\end{array}$ & 5 277-Glu, 292-Arg, 347-Tyr, \\
& & & \multicolumn{3}{c}{ 371-Arg, 406-Tyr } \\
\hline
\end{tabular}

Molecular docking was performed using AutoDock 4.2.6 at the active site of NA as used on validation process. For each test ligand, one pose with most negative $\Delta \mathrm{G}$ and lowest $\mathrm{K}_{\mathrm{i}}$ scores was selected as representative of the test ligand. The docking results of all test ligands were compared each other as shown in table $3 a$ and $3 b$. 
Table 3a. Docking result of NA receptors with all test ligand

\begin{tabular}{|c|c|c|c|c|c|c|}
\hline Parameters & DHA & HAL & HFL & HFR & BBR & CLB \\
\hline$\Delta \mathrm{G}(\mathrm{kcal} / \mathrm{mol})$ & -7.00 & -6.79 & -7.98 & -7.75 & -7.65 & -6.48 \\
\hline $\mathrm{K}_{\mathrm{i}}(\mu \mathrm{M})$ & 7.37 & 10.58 & 1.41 & 2.08 & 2.45 & 17.9 \\
\hline \multirow{25}{*}{$\begin{array}{l}\text { Amino acids } \\
\text { residues }\end{array}$} & - & 118-Arg & - & 118-Arg & 118-Arg & 118-Arg \\
\hline & - & 149-Val & - & - & 149-Val & 149-Val \\
\hline & 151-Asp & 151-Asp & - & 151-Asp & 151-Asp & - \\
\hline & 152-Arg & - & 152-Arg & 152-Arg & - & - \\
\hline & 178-Trp & - & 178-Trp & 178-Trp & - & - \\
\hline & - & - & 179-Ser & 179-Ser & - & - \\
\hline & 222-Ile & - & - & - & - & - \\
\hline & 224-Arg & - & 224-Arg & 224-Arg & - & - \\
\hline & 227-Glu & - & 227-Glu & 227-Glu & - & - \\
\hline & 246-Ser & - & - & - & - & - \\
\hline & 277-Glu & - & 277-Glu & 277-Glu & - & - \\
\hline & - & 292-Arg & 292-Arg & 292-Arg & - & - \\
\hline & - & 347-Tyr & - & - & - & 347-Tyr \\
\hline & - & - & - & - & - & 369-Asn \\
\hline & - & - & - & - & - & 370-Ser \\
\hline & - & 371-Arg & 371-Arg & 371-Arg & 371-Arg & 371-Arg \\
\hline & - & - & - & - & 403-Trp & 403-Trp \\
\hline & 406-Tyr & - & 406-Tyr & 406-Tyr & - & - \\
\hline & - & 427-Ile & - & - & 427-Ile & 427-Ile \\
\hline & - & - & - & - & 429-Gly & - \\
\hline & - & - & - & - & 430-Arg & - \\
\hline & - & 431-Pro & - & - & 431-Pro & 431-Pro \\
\hline & - & - & - & - & 432-Lys & 432-Lys \\
\hline & - & - & - & - & 433-Glu & - \\
\hline & - & - & - & - & 439-Thr & - \\
\hline $\begin{array}{c}\text { Numbers of } \\
\text { hydrogen bonds }\end{array}$ & 2 & 4 & 1 & 4 & 1 & 2 \\
\hline
\end{tabular}

Table 3b. Docking result of NA receptors with all test ligand

\begin{tabular}{|c|c|c|c|c|c|}
\hline Parameters & FLC & FRR & JRR & PMT & TNP \\
\hline$\Delta \mathrm{G}(\mathrm{kcal} / \mathrm{mol})$ & -8.12 & -7.25 & -6.88 & -6.66 & -7.70 \\
\hline \multirow[t]{11}{*}{$\mathrm{K}_{\mathrm{i}}(\mu \mathrm{M})$} & 1.11 & 4.84 & 9.13 & 13.22 & 2.25 \\
\hline & - & - & 118-Arg & 118-Arg & 118-Arg \\
\hline & - & - & - & - & 119-Glu \\
\hline & - & - & 149-Val & 149-Val & - \\
\hline & - & - & - & 151-Asp & 151-Asp \\
\hline & 152-Arg & - & - & - & 152-Arg \\
\hline & 178-Trp & - & - & - & - \\
\hline & 179-Ser & - & - & - & - \\
\hline & 222-Ile & - & - & - & 222-Ile \\
\hline & 224-Arg & - & - & - & 224-Arg \\
\hline & 227-Glu & - & - & - & 227-Glu \\
\hline Amino acids & 277-Glu & - & - & - & 277-Glu \\
\hline \multirow[t]{11}{*}{ residues } & 292-Arg & - & - & - & 292-Arg \\
\hline & - & 347-Tyr & 347-Tyr & 347-Tyr & - \\
\hline & - & - & 369-Asn & 369-Asn & - \\
\hline & - & - & 370-Ser & 370-Ser & - \\
\hline & 371-Arg & 371-Arg & 371-Arg & 371-Arg & 371-Arg \\
\hline & - & - & 403-Trp & 403-Trp & - \\
\hline & 406-Tyr & - & - & - & 406-Tyr \\
\hline & - & - & 427-Ile & 427-Ile & - \\
\hline & - & 431-Pro & 431-Pro & 431-Pro & - \\
\hline & - & 432-Lys & 432-Lys & 432-Lys & - \\
\hline & - & - & 439-Thr & - & - \\
\hline $\begin{array}{c}\text { Numbers of } \\
\text { hydrogen bonds }\end{array}$ & 2 & 2 & 2 & 1 & 3 \\
\hline
\end{tabular}


Compared to other ligands, fibleucin had the most negative $\Delta \mathrm{G}$ and the lowest $\mathrm{K}_{\mathrm{i}}$ thus had the highest affinity toward NA than other secondary metabolites of akar kuning. All ligands also provided negative scores of $\Delta \mathrm{G}$, indicated that interaction between NA and all ligands will occur spontaneously [14].
Although fibleucin had the highest affinity toward NA, the affinity was still lower than oseltamivir as comparison ligand. Pharmacophore optimization then performed to increase fibleucin affinity toward NA, as shown in table $4 . \quad$ Optimization was conducted based on deletion of pharmacophore

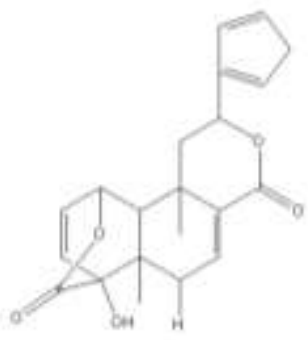


Fibleucin-6

(FLC-6)

All fibleucin derivatives were geometry optimized using Hartree-Fock method basis sets 6-311G. Docking was then performed again on all fibleucin derivatives toward NA using similar configurations with docking process before. The docking results of all fibleucin derivatives were compared each other as shown in table 5. Notice the significant increase in affinity of fibleucin-5 and decrease in fibleucin-4.

Table 5. Docking result of NA receptors with all fibleucin derivatives

\begin{tabular}{|c|c|c|c|c|c|c|}
\hline Parameters & FLC-1 & FLC-2 & FLC-3 & FLC-4 & FLC-5 & FLC-6 \\
\hline$\Delta \mathrm{G}(\mathrm{kcal} / \mathrm{mol})$ & -7.95 & -8.09 & -7.86 & -7.66 & -8.39 & -7.95 \\
\hline $\mathrm{K}_{\mathrm{i}}(\mu \mathrm{M})$ & 1.5 & 1.18 & 1.74 & 2.41 & 0.7106 & 1.49 \\
\hline & $\begin{array}{l}- \\
- \\
- \\
- \\
- \\
- \\
- \\
- \\
- \\
- \\
-\end{array}$ & $\begin{array}{l}- \\
- \\
- \\
- \\
- \\
- \\
- \\
- \\
- \\
- \\
-\end{array}$ & $\begin{array}{c}\text { 152-Arg } \\
\text { 178-Trp } \\
\text { 179-Ser } \\
\text { 222-Ile } \\
\text { 224-Arg } \\
\text { - } \\
246-\text { Ser } \\
276-\text { Glu } \\
277-\text { Glu } \\
\text { - } \\
294-\text { Asn }\end{array}$ & $\begin{array}{l}- \\
- \\
- \\
- \\
- \\
- \\
- \\
- \\
- \\
- \\
-\end{array}$ & $\begin{array}{l}- \\
- \\
- \\
- \\
- \\
- \\
- \\
- \\
- \\
- \\
-\end{array}$ & $\begin{array}{c}\text { 152-Arg } \\
\text { 178-Trp } \\
\text { 179-Ser } \\
222-\text { Ile } \\
224-\text { Arg } \\
\text { 227-Glu } \\
246-S e r \\
- \\
277-\text { Glu } \\
292-\text { Arg } \\
\text { 294-Asn }\end{array}$ \\
\hline $\begin{array}{l}\text { Amino acids } \\
\text { residues }\end{array}$ & $\begin{array}{c}\text { 347-Tyr } \\
\text { 369-Asn } \\
\text { 370-Ser } \\
\text { 371-Arg } \\
\text { 403-Trp } \\
- \\
427-\text {-Ile } \\
- \\
- \\
- \\
431-\text {-Pro } \\
432-\text {-Lys } \\
-\end{array}$ & $\begin{array}{c}\text { 347-Tyr } \\
\text { 369-Asn } \\
\text { 370-Ser } \\
\text { 371-Arg } \\
\text { 403-Trp } \\
- \\
427-\text { Ile } \\
- \\
- \\
- \\
431-\text {-Pro } \\
432-\text {-ys } \\
-\end{array}$ & $\begin{array}{c}347-\text { Tyr } \\
- \\
- \\
- \\
- \\
- \\
- \\
- \\
- \\
- \\
- \\
- \\
-\end{array}$ & $\begin{array}{c}\text { 347-Tyr } \\
- \\
- \\
\text { 371-Arg } \\
\text { 403-Trp } \\
\text { - } \\
\text { 427-Ile } \\
\text { 428-Arg } \\
\text { 429-Gly } \\
\text { 430-Arg } \\
\text { 431-Pro } \\
\text { 432-Lys } \\
\text { 433-Glu }\end{array}$ & $\begin{array}{c}\text { 347-Tyr } \\
- \\
- \\
\text { 371-Arg } \\
\text { 403-Trp } \\
\text { - } \\
\text { 427-Ile } \\
\text { - } \\
\text { 429-Gly } \\
\text { 430-Arg } \\
\text { 431-Pro } \\
\text { 432-Lys } \\
\text { 433-Glu }\end{array}$ & $\begin{array}{c}347-\mathrm{Tyr} \\
- \\
- \\
- \\
- \\
406-\mathrm{Tyr} \\
- \\
- \\
- \\
- \\
- \\
- \\
-\end{array}$ \\
\hline $\begin{array}{c}\text { Numbers of } \\
\text { hydrogen bonds }\end{array}$ & 1 & 1 & 2 & 0 & 1 & 1 \\
\hline
\end{tabular}

Further observation conducted to reveal the interaction between all fibleucin derivatives and oseltamivir as comparison ligand against NA active site by overlay the docking results. Interestingly, only fibleucin, fibleucin-3 and fibleucin-6 were docked into a similar position with oseltamivir, indicating most likely similar activity as NA inhibitor as oseltamivir [18].

The comparisons could be seen in figure 2 . 


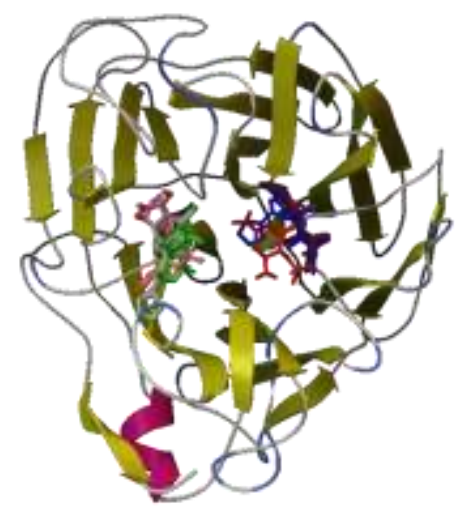

Figure 2. Comparison between docking results of fibleucin derivatives and oseltamivir against NA receptor (Red: oseltamivir; blue: fibleucin; orange: fibleucin-1; pink: fibleucin-2; violet: fibleucin-3; green: fibleucin-4; gray: fibleucin-5; brown: fibleucin-6)

\section{DISCUSSION}

All test ligands were sketched and geometry optimized with Hartree-Fock method with basis set 6-311G for geometry optimization. This method was ab initio approximation with relatively high confidence rate [10]. Docking validation was done with redocking method at the active site of NA receptor using AutoDock 4.2.6. All parameters were set to default protocol except for numbers of genetic algorithms runs which set to 100 runs to improve the accuracy of docking result [19]. Although almost, the result was still considered as valid for molecular docking purpose.

All test ligands provided negative scores of $\Delta \mathrm{G}$ in different ranges, with fibleucin had the most negative score. However, the score itself still below oseltamivir as co-crystal ligand. Pharmacophore optimization was then conducted not only to increase the affinity of fiblecuin but also to determine the main pharmacophore of fibleucin. The deletion method was selected to determine the main pharmacophore of fibleucin, where ligand with deleted pharmacophore resulting less affinity than fibleucin indicating their loss of affinity [20].

Interestingly, almost all derivatives show decreased affinity except fibleucin-5, indicating deletion of the hydroxyl group of fibleucin affects the affinity of fibleucin toward NA receptor. However, amino acids residues of fibleucin-5 much different with oseltamivir, indicating less-likely had same activity with oseltamivir. Important results were shown by ligand fibleucin-4, where the affinity decreased greater than another ligand. This result indicating that fibleucin-4, with deletion of pharmacophore ether group at position number 25 has lost it affinity against NA. This results also indicated that modification could be performed on several pharmacophores of fibleucin but ether group at position number 25 since it has important 
properties on the affinity of fibleucin against

NA receptor.

\section{REFERENCES}

[1]. Von Itzsein M. The war against influenza: Discovery and development of sialidase inhibitors. Nat Rev Drug Discov 6(12):967-74.

[2]. Pratama MR, Gusdinar T. Between artemisinin and derivatives with neuraminidase: a docking study insight. Asian J Pharm Clin Res 2017;10(8):3048.

[3]. Shen Z, Lou K, Wang W. New smallmolecule drug design strategies for fighting resistant influenza A. Acta Pharm Sin B 2015;5(5):419-30.

[4]. Setyowati R, Sudarsono, Setyowati EP. The effect of water-soluble stem extract kayu kuning (Arcangelisia flava L. Merr) on the growth inhibition of Candida albicans ATCC 10231 and Trichophyton mentagrophytes in vitro. Biology Medicine \& Natural Product Chemistry 2014;3(1):15-9.

[5]. Pratama MR. Akar kuning (Arcangelisia flava) sebagai inhibitor EGFR: Kajian in silico. Jurnal Farmagazine 2016;3(1):616.

[6]. Adcock SA, McCammon JA. Molecular dynamics: Survey of methods for simulating the activity of proteins. Chem Rev 2006;106(5):1589-615.

[7]. Kaserer T, Beck KR, Akram M, Odermatt A, Schuster D. Pharmacophore models and pharmacophore-based virtual screening: Concepts and Applications exemplified on hydroxysteroid dehydrogenases. Molecules 2015;20(12):22799-832.

[8]. Kroemer RT. Structure-based drug design: docking and scoring. Curr Protein Pept Sci 2007;8(4):312-28.

[9]. Meng XY, Zhang HX, Mezei M, Cui M. Molecular docking: a Powerful approach for structure-based drug discovery. Curr Comput Aided Drug Des 2011;7(2):14657.

[10]. Cosconati S, Forli S, Perryman AL, Harris R, Goodsell DS, Olson AJ. Virtual screening with autodock: Theory and practice. Expert Opin Drug Discov 2010;5(6):597-607.

[11]. O'Boyle NM, Banck M, James CA, Morley C, Vandermeersch T, Hutchison GR. Open Babel: an open chemical toolbox. J Cheminform 2011;3:33.

[12]. Morris GM, Huey R, Lindstrom W, Sanner MF, Belew RK, Goodsell DS, et al. AutoDock 4 and AutoDockTools 4: automated docking with selective receptor flexibility. J Comput Chem 2009;30(16):2785-91.

[13]. Russell RJ, Haire LF, Stevens DJ, Collins PJ, Lin YP, Blackburn GM, et al. The structure of $\mathrm{H} 5 \mathrm{~N} 1$ avian influenza neuraminidase suggests new opportunities for drug design. Nature 2006;443(7107):45-9.

[14]. Kontoyianni M, McClellan LM, Sokol GS. Evaluation of docking performance: comparative data on docking algorithms. J Med Chem 2004;47(3):558-65.

[15]. Bissantz C, Folkers G, Rognan D. Protein-based virtual screening of chemical databases 1. Evaluation of different docking/scoring combinations. J Med Chem 2000;43(25):4759-67.

[16]. Huang N, Shoichet BK, Irwin JJ. Benchmarking sets for molecular docking. J Med Chem 2006;49(23):6789801.

[17]. Kim R, Skolnick J. Assesment of Programs for ligand binding affinity prediction. J Comput Chem 2008;29(8):1316-31.

[18]. Narayanan MM, Nair CB, Sanjeeva SK, Rao PV, Pullela PK, Barrow CJ. Design of multiligand inhibitors for the swine flu H1N1 neuraminidase binding site. Adv Appl Bioinform Chem 2013;6:47-53

[19]. Arba M, Ihsan S, Ramadhan LO, Tjahjono DH. In silico study of Porphyrin-anthraquinone hybrids as CDK2 inhibitor. Comput Biol Chem 2017;67:9-14.

[20]. Lee CH, Huang HC, Juan HF. Reviewing ligand-based rational drug design: The search for an ATP synthase inhibitor. Int J Mol Sci 2011;12:5304-18. 